\title{
Psychosocial impact on puberphonic and effectiveness of voice therapy: A case report
}

\author{
${ }^{1}$ B. Bhattarail ${ }^{1}$ A. Shrestha ${ }^{2}$, Sunil Kumar Shah ${ }^{3}$
}

${ }^{1}$ Audiologist and Speech Therapist, Dept. of ENT, ${ }^{2}$ Lecturer, Dept. of ENT, ${ }^{3}$ Lecturer, Dept of Psychiatry

\begin{abstract}
Puberphonia is a rare disorder, in which the patient manifests higher register voice than others of their same age group. Manifestation is less in women than men. The prevalence is 1 in 900,000. Apart from impact on voice of patient, puberphonia also has impact on the psycho-social aspect. Different treatment modalities have been put forward in the past, many of which lack validity and EBP. Voice therapy has proven to be the most effective in the management of puberphonia.

Voice Handicap Index (VHI) is a tool for assessing the perceived handicap by the patient. VHI has 3 parts and overall score of 120 and individual subset has score of 40 each.
\end{abstract}

Result: Patient who received voice therapy obtained better score on the overall scale as well as on each subsets of the VHI.

Conclusion: The study concludes that the voice therapy not only improves the voice quality of the patient, but also improves the quality of life of the patient. The impact of voice disorder (puberphonia) is most prominent on the emotional section.

Keywords: Puberphonia, voice therapy, VHI.

\section{Introduction:}

Puberphonia is a disorder predominant on post-pubescent male (without known organic cause), who exhibit higher register than other male of the same age group. This disorder has also been observed in females, where the manifestation is very less, as females generally have higher register voice. This condition in female is known as "Juvenile Resonance Disorder" or "Little Girls’ Voice".

The high voice may be produced at the top of the chest register or in falsetto; this high voice is sometimes called mutational falsetto. It may be

Correspondences: Mr. Bibek Bhattarai

E-mail: bibeck.bhattarai@gmail.com associated with mild dysphonia and increased, effortful phonation. Vocal instability is often marked with extensive frequency swing.

Vocal impairment in male is generally of higher pitch i.e. above $200 \mathrm{~Hz}$, and sometimes observable with downward pitch breaks. Downward pitch break reveals the natural lower pitch level, which may be close to $110-125 \mathrm{~Hz}$.

\section{○ Aerodynamic characteristics:}

Possibly elevated then average airflow, if breathy voice accompanies higher pitch.

○ Acoustic characteristics:

Elevated in young men and women during or after pubertal changes. 
Prevalence of puberphonia is not well established. In one study by Banerjee (in Press), it was estimated that the prevalence of puberphonia, in India is about 1 in 900,000 , which was supported by other studies ${ }^{1}$. Juvenile Resonance Disorder is very rare as female generally have higher pitched voice ${ }^{2}$.

The normal course of disorder is high pitched, prepubescent voice, possibly accompanying downward pitch break (in males) persisting after puberty.

Negative social reactions commonly occur, affecting overall social and vocational goals.

\section{Impact of voice disorder:}

The impact of voice disorder varies greatly from person to person. Occupation, environment, family members and overall personality are all the variables that can affect the way voice disorder affects a specific person. In general, people with puberphonia tend to encounter problems that include psychological, emotional, social and professional related difficulty ${ }^{3}$. Recently there has been increased interest in the HRQOL, and a research conducted by Wilson et. al. has emphasized the importance of including QOL measure in an otolaryngologic and voice assessment ${ }^{4}$.

\section{Voice Handicap Index:}

It was developed and validated by Jacobson, Johnson, Grgnalski, Silbergleit and Beginner in 1997. Initially it was developed to fill the requirement of patient's outcome with emphasis on patient's physical, emotional and functional changes as the treatment progresses. First version of VHI had 85 items, which was then reduced to form 30 item scale as VHI-30, which is the most popular scale used in both clinics and research ${ }^{5}$.
Each sub-section of VHI is weighs a score of 40, which gives total of 120 . A VHI score 0 to 30 represents low scores indicating that there is a minimal amount of handicap associated with the voice disorder. A score of 31 to 60 denotes a moderate amount of handicap due to voice problem. A VHI score from 60 to 120 represents significant and serious amount of handicap due to voice problem and are often seen in patient with new onset vocal fold paralysis or severe vocal fold scarring 6 .

Rosen et. al. (2000) reported that Voice Handicap Index as a useful instrument to monitor the treatment efficacy for wide range of voice disorders ${ }^{7 \& 8}$. VHI is also used to assess the effect voice disorder has on patients' daily living ${ }^{5}$. The overall VHI score, as well as the percentage change between VHI scores preto post-intervention, and scores on the individual subscales of VHI can be important for assessing treatment option and treatment outcome ${ }^{7}$.

\section{Pathophysiology:}

In infants, the laryngo-tracheal complex lies at a higher level. It descends rapidly during puberty in males. The larynx becomes larger \& unstable. Also the brain is more accustomed to infant voice. The boy may hence continue to use a high pitched voice or it may break into higher and lower pitches. Other causes can be strong feminine identification, desire to maintain the childhood soprano singing voice ${ }^{9}$.

\section{Objective:}

To understand the psycho-social impact on puberphonics before and after treatment (voice therapy). Also to demonstrate the efficacy of voice therapy on puberphonic, in conjunction with manual digital manipulation of muscles of larynx. 


\section{Rationale:}

Apart from impact on voice of the puberphonic, this disorder also has impact on social and psychological level. The case history generally concerns about the level of psychological impact of the patient, but research has not yet addressed the change in the psycho-social behavior of the patient after the success of voice therapy.

There have been researches on the efficacy of voice therapy for puberphonics, which has shown to be effective both in term of validity and Evidence Based Practice (EBP). This study is conducted to see the outcome in context of Nepal, as there is lack of awareness about the disorder and also the treatment outcome of it as well.

It has also been noted that the patient with voice disorder (especially puberphonic) has a tendency to be left out not due to how other people behave, instead due to the inferiority of oneself. Hence this study also takes on to see the outcome of voice therapy on that aspect. For which the emotional aspect of VHI will be used extensively, both before and after therapy.

Also there has been very little research, as only 8 researches (as shown on pub-med search) have been published so far since 1983. And also the available studies lack validity ${ }^{10}$.

\section{Methodology:}

20 year male attending voice therapy at college of medical sciences was selected for the preliminary study after medical and voice evaluation. Patient was asked to fill out the questionnaire of $\mathrm{VHI}$ before the treatment and after the successful restoration of voice (acceptable). Therapy was carried out at 2 sessions per week for 2 months. The evaluation will be based on the 3 parameters of VHI as Physical, Functional and Emotional. The overall score will be compared to see the changes in perceived impact of disorder. The score on emotional section will be considered for psychosocial changes after the treatment.

Treatment included:

$\square$ Digital manipulation of muscles of larynx.

$\square$ Pitch modulation.
○ Sliding
○ Twang
$\circ$ Rising
○ Prill
o Siren
○ Humming

$\square$ Pitch stabilization

$\square$ Other vegetative exercises as

○ Breathing exercise

- Abdominal breathing

$\nabla$ Counseling

\section{Review of literature:}

In a study by Murry and Rosen, the VHI was used to assess changes in the degree of handicap patients experience following voice treatment in which it was demonstrated that patients from different diagnostic groups (unilateral vocal cord paralysis, muscle tension dysphonia, and vocal cord polyp or vocal cord cyst) showed decrease in average VHI score following treatment. The study suggests that while the absolute score on VHI is important, the percentage of change between the pre-treatment and posttreatment score is the more critical measure when assessing treatment outcome ${ }^{7}$.

VHI has been proven to be a valuable tool in assessing self perceived handicap in a diverse population of voice patients. It has also proved to be effective in the evaluation of treatment outcome in wide range of voice disorders ${ }^{8}$. 


\section{Result:}

The scores when compared to the pre and post therapy showed marked differences on the overall score as well as on the individual subsets of VHI. The score before therapy was 94 (out of 120) on overall score and on each subsets it was as follows; emotional 35 (out of 40), functional 26 (out of 40) and on physical section 33 (out of 40). Overall rating of patients' voice within the period of 2 weeks was Poor.

The scores after the completion of the therapy were 6 on the overall score. On the subsection it was as follows; emotional 0 (out of 40), functional 1 (out of 40) and on physical section 5 (out of 40). The overall rating of voice in the period of 2 week was very good.

\section{Discussion:}

The higher scores on the overall and each subsection of the VHI conclusively prove that voice disorder affects the person's psycho-social life as well as his quality of life. The study also opened the door to other aspect of viewing puberphonic as; the maximum impact is seen on the emotional aspect of the VHI, which imposes that the management should aim more at the emotional aspect of the patient.

Change in the scores from pre to post therapy indicates that voice therapy is the effective tool for management of puberphonics, which is supported by the studies form past ${ }^{10 \& 11}$.

Earlier researches have equivocally stated the benefit as well as shortcoming of voice therapy for dysphonics, as MacKenzie et. $\mathrm{al}^{11}$ has concluded, in a longitudinal study, that voice therapy improves voice; but no improvement on QOL. Other studies as Rosen et. al. (1995 and 2005) indicated that there is improvement of QOL based on HR-QOL scales.
This study also supports the findings of earlier researches, wherein the focus has been only on puberphonics, rather than on broader spectrum as dysphonics. We have tried to explore primarily the psychosocial impact on puberphonic and secondarily efficacy of voice therapy. Though there have not been much researches on the psychosocial impact, there has been research on the efficacy of voice therapy ${ }^{10}$.

Review of literature shows that different techniques has been used in treatment of puberphonia, as surgical and laryngoscope procedure, both of which has poor validity/EPB ${ }^{1 \& 12}$. On the other hand, voice therapy has shown good validity and $\mathrm{EBP}^{10 \& 13}$.

As the prevalence of case is less, it is difficult to assess the incidence and treatment outcome ${ }^{10}$.

\section{Conclusion and Future Directions:}

The study concludes that the voice therapy is the most effective im management of puberphonia which is supported by earlier studies as well ${ }^{10 \& 13}$, and with successful completion of voice therapy, the patients' psychosocial level also gets improved; which in turn improves the quality of life. Also the VHI is handy tool for assessment as well as monitoring the progress of the therapy, which is perceived by the patient himself. Since this is the pilot study, it lacks the number of patients for generalizing the results, so the study can be carried out with greater population.

\section{Acknowledgement:}

We would like to thank our H.O.D Dr. N.S. Reddy for allowing us to carry out this study at the department. Also we would like to thank the patient for their participation in the study. 
B. Bhattarai et al. Psychosocial impact on puberphonic and effectiveness of voice therapy: A case report

\section{References:}

1. Pau H, Murty G.E. (2001). First case of surgically corrected puberphonia. The Journal of Laryngology \& Otology, 115, 60-1.

2. Hedge M.N. (2001). Introduction to communicative disorders, $3^{\text {rd }}$ Ed. Austin, Tx: Pro-Ed.

3. Scott S., Wilson J.A., Robinson K., et al Patient reported problems associated with dysphonia. Clinical Otolaryngology 1997; 22:37-40.

4. Wilson J., Deary I., Millar A. et al The quality of life impact of dysphonia. Clinics of Otolaryngology, 27(3), 179-82.

5. Jacobson B., Johnson A., Grywalski C., et al The Voice Handicap Index (VHI): Development and Validation. American Journal of Speech-Language Pathology, $6(3), 66-9$

6. Hedge M., Achala C., Bhat S. (2009). Voice Handicap index- A comparison of Clinician's Ratings and Self Rating by Individuals with dysphonia. JAIISH, 28, 2530.

7. Rosen C.A., Murry T., Zinn A. et al. Voice Handicap Index change following treatment of voice disorder. Journal of voice, 14(4), 619-23.
8. Beginner M., Ahuja A.S., Gardner G.,.Assessing outcomes for dysphonic patients. Jourmal of Voice, 12 (4), 540-50.

9. Stemple J.C., Glaze L.E., Klaben B.G. (2000). Clinical Voice Pathology: Theory and Management, $3^{\text {rd }}$ Ed. Clifton Park, NY: Delmar Cengage Learning.

10. Dagli M., Sati I., Acar A., et al.(2008) Mutational Falsetto: Intervention outcomes in 45 patients. The journal of Laryngology \& Otology, 122, 277-81.

11. MacKenzie K., Millar A., Wilson J.A., et al. Is voice therapy an effective treatment for dysphonia? A randomized controlled trial. British Medical Journal, $323,1-6$.

12. Vaidya S. Vyas G. (2006). Puberphonia: A Novel approach to treatment. Indian Journal of Otolaryngology and Head and Neck surgery, 58.

13. Lim J.Y., Lim S.E., Choi S.H., et al Clinical characteristic \& voice analysis of patients with mutational dysphonia: Clinical significance of diplophonia and closed quotients. Journal of voice, 21,12 . 


\section{Voice Handicap Index:}

\section{Part-I: Functional}

1. My voice makes it difficult for people to hear me.

$\begin{array}{lllll}0 & 1 & 2 & 3 & 4\end{array}$

2. People have difficulty understanding me in a noise room.

$\begin{array}{lllll}0 & 1 & 2 & 3 & 4\end{array}$

3. My family has difficulty hearing me when I call them throughout the house. $\quad \begin{array}{lllll}0 & 1 & 2 & 3 & 4\end{array}$

4. I use the phone less often than I would like to.

5. I tend to avoid groups of people because of my voice.

$\begin{array}{lllll}0 & 1 & 2 & 3 & 4\end{array}$

6. I speak with friend, neighbors, or relatives less often because of my voice.

$\begin{array}{llllll}0 & 1 & 2 & 3 & 4\end{array}$

7. People ask me to repeat myself when speaking face to face.

$\begin{array}{lllll}0 & 1 & 2 & 3 & 4\end{array}$

8. My voice difficulties restrict personal and social life.

$\begin{array}{lllll}0 & 1 & 2 & 3 & 4\end{array}$

9. I feel left out of conversations because of my voice problem.

$\begin{array}{lllll}0 & 1 & 2 & 3 & 4\end{array}$

10. My voice problem causes me to lose income.

\section{Part-II: Physical}

1. I run out of air when I talk.

$\begin{array}{lllll}0 & 1 & 2 & 3 & 4\end{array}$

2. The sound of my voice varies throughout the day.

$\begin{array}{lllll}0 & 1 & 2 & 3 & 4\end{array}$

3. People as, "What is wrong with your voice?"

$\begin{array}{lllll}0 & 1 & 2 & 3 & 4\end{array}$

4. My voice sounds creaky and dry.

$\begin{array}{lllll}0 & 1 & 2 & 3 & 4\end{array}$

5. I feel as though I have to strain to produce voice.

$\begin{array}{lllll}0 & 1 & 2 & 3 & 4\end{array}$

6. The clarity of my voice is unpredictable.

$\begin{array}{lllll}0 & 1 & 2 & 3 & 4\end{array}$

7. I try to change my voice to sound different.

$\begin{array}{lllll}0 & 1 & 2 & 3 & 4\end{array}$

8. I use a great deal of effort to speak.

$\begin{array}{lllll}0 & 1 & 2 & 3 & 4\end{array}$

9. My voice sounds worse in the evening.

$\begin{array}{lllll}0 & 1 & 2 & 3 & 4\end{array}$

10. My voice "gives out" on me in the middle of speaking.

$\begin{array}{lllll}0 & 1 & 2 & 3 & 4\end{array}$

\section{Part-III: Emotional}

1. I am tensed when talking to others because of my voice.

$\begin{array}{lllll}0 & 1 & 2 & 3 & 4\end{array}$

2. People seem irritated with my voice.

$\begin{array}{lllll}0 & 1 & 2 & 3 & 4\end{array}$

3. I find that other people don't understand my voice problem.

$\begin{array}{lllll}0 & 1 & 2 & 3 & 4\end{array}$

4. My voice problem upsets me.

$\begin{array}{lllll}0 & 1 & 2 & 3 & 4\end{array}$

5. I am less outgoing because of my voice problem.

$\begin{array}{lllll}0 & 1 & 2 & 3 & 4\end{array}$

6. My voice makes me feel handicapped.

$\begin{array}{lllll}0 & 1 & 2 & 3 & 4\end{array}$

7. I feel annoyed when people ask me to repeat.

$\begin{array}{lllll}0 & 1 & 2 & 3 & 4\end{array}$

8. I feel embarrassed when people ask me to repeat.

$\begin{array}{lllll}0 & 1 & 2 & 3 & 4\end{array}$

9. My voice makes me feel incompetent.

$\begin{array}{lllll}0 & 1 & 2 & 3 & 4\end{array}$

10. I am ashamed of my voice problem.

$\begin{array}{lllll}0 & 1 & 2 & 3 & 4\end{array}$

The overall quality of my voice during last 2 weeks has been (please circle):
Poor
Fair
Good
Very good
Excellent 